\title{
CRITICAL VELOCITY OF CONTROLLABILITY OF SLIDING FRICTION BY NORMAL OSCILLATIONS IN VISCOELASTIC CONTACTS
}

\author{
UDC 539.3
}

\author{
Mikhail Popov ${ }^{1,2,3}$ \\ ${ }^{1}$ Tomsk Polytechnic University, Tomsk, Russia \\ ${ }^{2}$ Technische Universität Berlin, Berlin, Germany \\ ${ }^{3}$ Tomsk State University, Tomsk, Russia
}

\begin{abstract}
Sliding friction can be reduced substantially by applying ultrasonic vibration in the sliding plane or in the normal direction. This effect is well known and used in many applications ranging from press forming to ultrasonic actuators. One of the characteristics of the phenomenon is that, at a given frequency and amplitude of oscillation, the observed friction reduction diminishes with increasing sliding velocity. Beyond a certain critical sliding velocity, there is no longer any difference between the coefficients of friction with or without vibration. This critical velocity depends on material and kinematic parameters and is a key characteristic that must be accounted for by any theory of influence of vibration on friction. Recently, the critical sliding velocity has been interpreted as the transition point from periodic stick-slip to pure sliding and was calculated for purely elastic contacts under uniform sliding with periodic normal loading. Here we perform a similar analysis of the critical velocity in viscoelastic contacts using a Kelvin material to describe viscoelasticity. A closed-form solution is presented, which contains previously reported results as special cases. This paves the way for more detailed studies of active control of friction in viscoelastic systems, a previously neglected topic with possible applications in elastomer technology and in medicine.
\end{abstract}

Key Words: Active Control of Friction, Ultrasonic Vibration, Viscoelastic Contact, Critical Velocity

Received October 22, 2016 / Accepted November 30, 2016

Corresponding author: Mikhail Popov

Technische Universität Berlin, Str. des 17. Juni 135, 10623 Berlin

E-mail:m@popov.name 


\section{INTRODUCTION}

The reduction of static and sliding friction by ultrasonic oscillation in various directions is a well-known phenomenon with many applications ranging from wire drawing and press forming, stabilization of system dynamics, as in brake squeal suppression, and production of directed motion, as in ultrasonic motors and linear actuators. The effect has been studied for several decades, both experimentally and theoretically. Among the proposed explanations, microscopic theories have historically been prevalent. E.g. Zaloj et al. [1] suggest that the effect may be due to the dilatation caused by sliding. V. Popov et al. point to the possible importance of the microscopic interaction potential [2]. Although plausible, microscopic models could never achieve good, quantitative correspondence between theoretical predictions and experimental results, e.g. [3]. Opposite to that stand purely macroscopic models, which explain the phenomenon using macroscopic contact mechanics or system dynamics. Several system configurations have been considered from that perspective $[3,4,5]$ and it was found that the macroscopic models can describe the observed behavior of the systems without fitting parameters. This result is in fact somewhat surprising, considering that these macroscopic theories assume a constant microscopic coefficient of friction and a friction law of the form $F_{f}=\mu_{0} F_{n}$. When the average force of friction is determined by integrating the force of friction over time (or integrating stress over time and contact area) and dividing by the integral of the normal force, the direct proportionality of the assumed law of friction will insure that the integrals of normal force will cancel out, with the end result that the average coefficient of friction $\bar{\mu}$ must always be equal to $\mu_{0}$. This reasoning, however, is subtly flawed, in that it assumes sliding in one direction with a nonzero velocity. It is also possible for the body to temporarily cease motion (e.g. due to increasing normal force or more complicated reasons relating to system dynamics). During such stick phases, the law of friction needs to be written in its static form: $F_{f} \leq \mu_{0} F_{n}$. Note the less than or equal in this formula, which breaks the proportionality and allows $\bar{\mu}$ to be less than $\mu_{0}$. To the author's knowledge, the possibility that the influence of normal oscillations on sliding friction may be explained entirely by the presence of intermittent stick phases has not been made explicit before the publication of the two part-study $[6,7]$. In these papers, the stick-induced reduction of friction force was studied in a displacementcontrolled setting with and without in-plane system dynamics. Although a closed-form solution for the actual force of friction under the action of normal vibrations does not exist in either case, it has turned out to be possible to calculate the critical velocity $v_{c}$ for a broad class of problems. This critical velocity refers to the maximum sliding velocity, above which vibration no longer has any influence on friction (at a given frequency and amplitude). This is illustrated in Fig. 1, which qualitatively describes the behavior of the average coefficient of friction, as it increases from its static value to $\mu_{0}$ with increasing sliding velocity. In the theory presented in [6] it was argued that this critical velocity is related to the disappearance of stick in the contact. Also in [6], the following expression was obtained for the critical velocity in an entirely displacement-controlled system:

$$
v_{c}=\mu_{0} \omega \Delta u_{z} \frac{E^{*}}{G^{*}},
$$


where $\omega \Delta u_{z}$ is the amplitude of velocity oscillation and $E^{*} / G^{*}$ is the ratio of the normal and tangential stiffness of the contact (the so-called Mindlin-ratio). Since this ratio is generally of the order of unity, one can roughly say that the critical sliding velocity is equal to the maximum velocity in the normal direction (due to the oscillation) times the microscopic coefficient of friction. This critical velocity also enters into the primary dimensionless parameter characterizing the behavior of the system, which makes accurate analysis of this quantity doubly important.

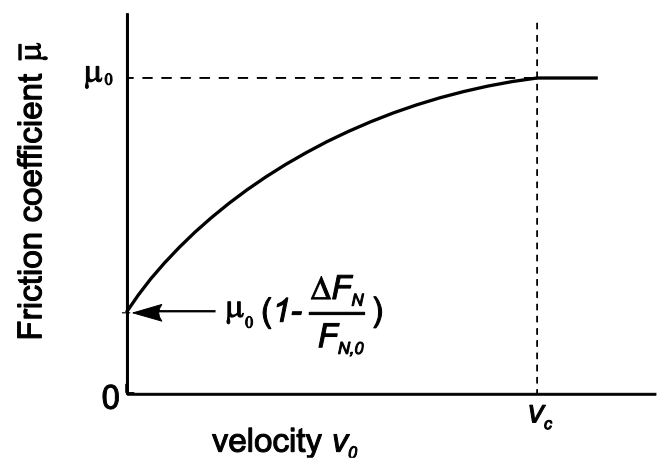

Fig. 1 Qualitative dependence of the average coefficient of friction (COF) on sliding velocity under action of normal oscillations. Of particular interest are the "static COF" at zero velocity, the monotonous increase of the COF with increasing sliding velocity and the critical velocity of controllability, above which the average COF is equal to the microscopic $\mathrm{COF}, \mu_{0}$, with or without oscillations.

If the model is augmented with a system spring and a contact mass, thus enabling inplane system dynamics, the expression for the critical velocity becomes [7]:

$$
v_{c}=\mu_{0} \omega \Delta u_{z} \frac{k_{z, c}}{k_{x, c}} \frac{\left|k_{x, c}+k_{x}-m \omega^{2}\right|}{\left|k_{x}-m \omega^{2}\right|},
$$

where $k_{x, c}$ and $k_{z, c}$ are the tangential and normal stiffness of the contact (in this model, the contact stiffness is assumed to be constant), $k_{x}$ is the tangential stiffness of the surrounding system and $m$ is the mass of the sliding body. The only difference compared to Eq. (1) is the additional dependence on the two natural frequencies of the system. Indeed, if $k_{x}$ tends to infinity, Eq. (2) reduces to the previous result. Another notable feature is the presence of two resonant frequencies, in particular $\omega=\sqrt{k_{x}} / m$ where $v_{c}$ becomes infinite. Numerical experiments show that in this case, the coefficient of friction reaches a plateau (which is less than $\mu_{0}$ ) at fairly low sliding velocities and does not change thereafter. For the full analysis, the reader is referred to $[6,7]$.

In the present paper these previous results are extended to also include viscoelastic contacts. Active control of friction and system stability seems to be an underexplored topic when viscoelastic contacts are concerned, despite many possible applications in conjunction with the ubiquitous use of elastomers and the rising demands placed on devices in contact with biological tissues in medical technology. With this paper we 
would like to begin establishing a quantitative framework for the analysis of viscoelastic friction under oscillation, by proposing that the same methods used in $[6,7]$ can be applied in viscoelastic contacts in order to calculate the critical velocity in closed form.

\section{MODEL AND ANALYSIS}

\subsection{Formulation of the model}

The model that will be analyzed in this paper is very similar to the one presented in [7]. It consists of a mass $m$ that is pulled with a constant velocity $v_{0}$ through a system spring with a constant stiffness $k_{x}$ (see Fig. 2). In addition, a displacement-controlled harmonic oscillation is imposed in the direction normal to the plane. The oscillation is defined by:

$$
u_{z}=u_{z, 0}+\Delta u_{z} \cos \omega t
$$

where $u_{z}$ is the coordinate of the body in the normal direction, $u_{z, 0}$ the mean indentation depth, $\Delta u_{z}$ the oscillation amplitude and $\omega$ the frequency. The body is connected to the substrate through a contact point, in which Amontons' law of friction with a constant coefficient of friction $\mu_{0}$ is assumed. The main difference is that the contact is not elastic but viscoelastic and characterized not only by the constant tangential and normal spring stiffness $k_{x, c}$ and $k_{z, c}$, but also by the dynamic viscosities $\gamma_{x, c}$ and $\gamma_{z, c}$. This corresponds to the Kelvin material, the simplest model of viscoelasticity. The relevant dynamics of the resulting system is confined to the sliding plane and is characterized by $u_{x}$, the position of the body and $u_{x, c}$, the position of the contact point.

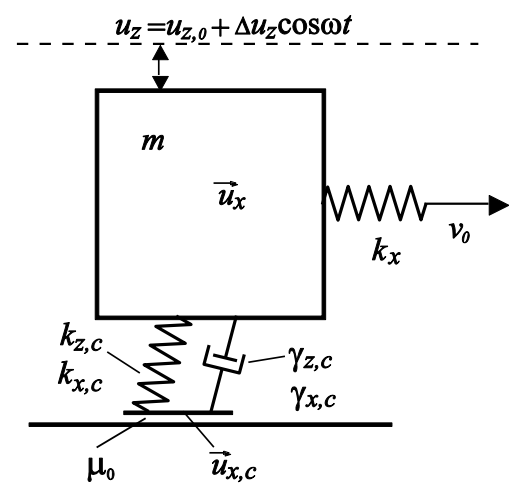

Fig. 2 Schematic representation of the considered system, consisting of a mass, a system spring and a viscoelastic contact with the sliding plane.

\subsection{Analysis of the model}

\subsubsection{Normal force}

The normal force in the spring-damper combination is given by:

$$
F_{N}=k_{z, c} u_{z}+\gamma_{z, c} \dot{u}_{z}=k_{z, c}\left(u_{z, 0}+\Delta u_{z} \cos \omega t\right)-\gamma_{z, c} \omega \Delta u_{z} \sin \omega t .
$$


To ensure that the body is always in contact with the plane, the normal force must always remain positive. This is the case if:

$$
k_{z, c} u_{z, 0}>\Delta u_{z} \sqrt{k_{z, c}{ }^{2}+\gamma_{z, c}{ }^{2} \omega^{2}} .
$$

Only this "non-jumping" case is considered in the following.

The static force of friction (the force at zero sliding velocity) can be calculated easily by noting that, according to Eq. (4), the amplitude of the oscillation of the normal force is equal to:

$$
\Delta F_{N}=\Delta u_{z} \sqrt{k_{z, c}^{2}+\gamma_{z, c}^{2} \omega^{2}} .
$$

The static force of friction is equal to the minimal normal force during an oscillation cycle, multiplied with the coefficient of friction:

$$
F_{s}=\mu_{0}\left(F_{N, 0}-\Delta F_{N}\right)=\mu_{0}\left(k_{z, c} u_{z, 0}-\Delta u_{z} \sqrt{k_{z, c}{ }^{2}+\gamma_{z, c}{ }^{2} \omega^{2}}\right) .
$$

\subsubsection{Tangential movement}

Under the assumption that the immediate contact point is always in the sliding state, the equation of motion of mass $m$ reads:

$$
m \ddot{u}_{x}=k_{x}\left(v_{0} t-u_{x}\right)-\mu_{0} F_{N} .
$$

The equilibrium condition for the "foot point" of the spring-damper combination reads:

$$
k_{x, c}\left(u_{x}-u_{x, c}\right)+\gamma_{x, c}\left(\dot{u}_{x}-\dot{u}_{x, c}\right)=\mu_{0} F_{N},
$$

where $F_{N}$ is given by Eq. (4).

Equation (8), after inserting Eq. (4) on the right hand side, can be easily solved with respect to $u_{x}$ :

$$
u_{x}=v_{0} t-\mu_{0} \frac{k_{z, c}}{k_{x}} u_{z, 0}+\frac{\mu_{0} \Delta u_{z}}{m \omega^{2}-k_{x}}\left(k_{z, c} \cos \omega t-\gamma_{z, c} \omega \sin \omega t\right) .
$$

In our analysis we assume that the material of the contacting elastomer body is isotropic, with a constant (frequency-independent) Poisson number. Under these conditions, we have:

$$
\frac{\gamma_{x, c}}{\gamma_{z, c}}=\frac{k_{x, c}}{k_{z, c}} .
$$

Equation (9) can also be solved with respect to $\left(u_{x}-u_{x, c}\right)$ :

$$
u_{x}-u_{x, c}=\mu_{0} \frac{k_{z, c}}{k_{x, c}}\left(u_{z .0}+\Delta u_{z} \cos \omega t\right) .
$$

From Eqs. (10) and (12) we can first determine $u_{x, c}$ : 
$u_{x, c}=v_{0} t-\mu_{0} k_{z, c} u_{z, 0}\left(\frac{1}{k_{x}}+\frac{1}{k_{x, c}}\right)+\frac{\mu_{0} \Delta u_{z}}{m \omega^{2}-k_{x}}\left(k_{z, c} \cos \omega t-\gamma_{z, c} \omega \sin \omega t\right)-\mu_{0} \frac{k_{z, c}}{k_{x, c}} \Delta u_{z} \cos \omega t$

and finally $\dot{u}_{x, c}$ :

$$
\begin{aligned}
& \dot{u}_{x, c}=v_{0}+\frac{\mu_{0} \Delta u_{z}}{m \omega^{2}-k_{x}}\left(-k_{z, c} \omega \sin \omega t-\gamma_{z, c} \omega^{2} \cos \omega t\right)+\mu_{0} \frac{k_{z, c}}{k_{x, c}} \Delta u_{z} \omega \sin \omega t \\
& =v_{0}-\frac{\mu_{0} \Delta u_{z}}{m \omega^{2}-k_{x}} \gamma_{z, c} \omega^{2} \cos \omega t+\mu_{0} \Delta u_{z} \omega \frac{k_{z, c}}{k_{x, c}} \frac{-k_{x, c}-k_{x}+m \omega^{2}}{\left(m \omega^{2}-k_{x}\right)} \sin \omega t
\end{aligned}
$$

The critical velocity of controllability is given by the condition that the amplitude of the oscillating part of this solution becomes equal to constant sliding velocity $v_{c}$ :

$$
v_{c}=\frac{\mu_{0} \Delta u_{z} \omega}{\left|m \omega^{2}-k_{x}\right|} \sqrt{\left(\gamma_{z, c} \omega\right)^{2}+\left(\frac{k_{z, c}}{k_{x, c}} \cdot\left(m \omega^{2}-k_{x, c}-k_{x}\right)\right)^{2}} .
$$

Note that the critical velocity depends on the oscillation amplitude but not on the average indentation.

In the limit of a very stiff system spring, $k_{x} \rightarrow \infty$, the critical velocity, Eq. (15), is reduced to Eq. (1), which thus appears to be valid independently of the viscoelastic properties of the medium. According to the Method of Dimensionality Reduction (MDR) [8], any rotationally symmetric contact can be equivalently represented by a model consisting of a series of independent springs (note that an equivalent one-dimensional model can in fact be constructed for almost arbitrary, e.g. rough, contacts, although there may be no closed-form mapping rule in the general case). As has been argued in [6], the existence an equivalent model with uncoupled spring elements, together with the indentation-independence of Eq. (15), implies that the obtained result in Eq. (15) is valid not only for the simple considered model with a single spring-damper combination, but also for quite general contacts (so long as the amplitude of oscillation remains small).

\section{CONCLUSION}

While the details of the influence of oscillation on friction may be very complicated at intermediate sliding velocities [7], there are still two simple and nearly universal (except in resonant cases) characteristic points: First, the velocity-dependences all start from the static value at vanishing velocity. Second, the coefficient of friction increases monotonically (again, barring exceptional system-dynamical circumstances) until it reaches the microscopic value at some critical velocity. These two points, the static coefficient of friction, and the critical velocity of controllability of friction, are the most important characteristics of any oscillating frictional system. It so happens that both of these points can be determined analytically for very general classes of contacts with and without system dynamics.

In the present paper, the critical velocity of controllability was determined for the simplest possible viscoelastic rheology (Kelvin body) and the simplest possible contact geometry (contact with constant contact stiffness, e.g. cylindrical punch). Eq. (15) provides 
an explicit analytical solution. Even under these simple assumptions, the critical velocity depends on almost all system and loading parameters: the local coefficient of friction $\mu_{0}$, mass $m$ of the system, the stiffness of the contact and of the system, the frequency of oscillations, the damping coefficient of the contact, and on the amplitude of oscillations. However, it does not depend on absolute indentation, which permits easy generalization to more realistic contact geometry.

Further, in the case of displacement-controlled horizontal movement (corresponding to an infinitely stiff surrounding system, which eliminates system dynamics in the contact) it was found that the critical velocity is given by Eq. (1), without dependence on the rheological properties of the contact: only the ratio of the contact stiffness (Mindlin ratio) appears in the expression for this critical velocity.

In the future, the critical velocity could also be considered for materials with more general rheology. The Method of Dimensionality Reduction [8] provides a natural theoretical framework for this and for further generalizations to arbitrary contact geometries and loading histories.

Acknowledgements: The author would like to thank V. L. Popov and A. E. Filippov for inspiring discussions concerning the present work.

\section{REFERENCES}

1. Zaloj, V, Urbakh, M, Klafter, J., 1999, Modifying friction by manipulating normal response to lateral motion. Physical review letters 82(24), pp. 4823, 1999.

2. Popov, VL, Starcevic, J, Filippov, AE., 2010, Influence of ultrasonic in-plane oscillations on static and sliding friction and intrinsic length scale of dry friction processes. Tribology Letters, 39 (1), 25-30, 2010.

3. Teidelt, E, 2015, Oscillating contacts: friction induced motion and control of friction, Dissertation, TU Berlin, Germany, $151 \mathrm{p}$

4. Milahin, N., Starcevic, J., 2014, Influence of the normal force and contact geometry on the static force of friction of an oscillating sample. Physical Mesomechanics, 17(3), pp. 84-8.

5. Milahin, N., Li, Q., 2016, Friction and wear of a spherical indenter under influence of out-of-plane ultrasonic oscillations. Physical Mesomechanics, 19(2), pp. 149-153.

6. Popov, M., Popov, VL, Popov, NV, 2016, Reduction of friction by normal oscillations. I. Influence of contact stiffness, arXiv:1611.07017.

7. Mao, X, Popov, VL, Starcevic, J., Popov, M, 2016, Reduction of friction by nor-mal oscillations. II. In-plane system dynamics, arXiv:1611.07018.

8. Popov, VL., Heß, M. 2015, Method of dimensionality reduction in contact mechanics and friction, Springer, Berlin, Heidelberg, $265 \mathrm{p}$. 